Editorial

\title{
Novel Bituminous Materials for Sustainable Pavements
}

\author{
José Norambuena-Contreras $\mathbb{D}^{1},{ }^{1}$ Lily Poulikakos, ${ }^{2}$ Hassan Baaj $\mathbb{D},{ }^{3}$ and Quantao Liu $\mathbb{D}^{4}$ \\ ${ }^{1}$ LabMAT, Department of Civil and Environmental Engineering, University of Bío-Bío, Concepción, Chile \\ ${ }^{2}$ Empa-Swiss Federal Laboratories for Materials Science and Technology, Dübendorf, Switzerland \\ ${ }^{3}$ Department of Civil and Environmental Engineering, University of Waterloo, Waterloo, Canada \\ ${ }^{4}$ Wuhan University of Technology, State Key Laboratory of Silicate Materials for Architectures, Wuhan, China \\ Correspondence should be addressed to José Norambuena-Contreras; jnorambuena@ubiobio.cl
}

Received 20 December 2019; Accepted 17 January 2020; Published 19 February 2020

Copyright ( $) 2020$ José Norambuena-Contreras et al. This is an open access article distributed under the Creative Commons Attribution License, which permits unrestricted use, distribution, and reproduction in any medium, provided the original work is properly cited.

Bituminous materials are complex viscoelastic composites mainly used for road and airport pavement construction. Numerous advances on novel bituminous materials for sustainable pavements have been developed over the last years, turning this topic into an emerging field of study. The studies include several areas: characterization and modelling of bituminous materials, multiphase behaviour of bituminous materials, asphalt mixtures modified with phase change materials, self-healing of asphalt pavements, and valorisation of waste for roads.

This special issue presents a selection of thirteen recent research studies mainly focused on the multiscale characterization and numerical modelling of bituminous materials including modified asphalt mixtures, mastics and bituminous binders, recycling and valorisation of waste materials for asphalt pavements, as well as innovations in more sustainable bituminous materials development and on-site application. Scientific contributions from Canada, China, USA, Czech Republic, Republic of Korea, Italy, France, and Spain were received.

This special issue opens with the study conducted by A. Hamid et al. They have developed a laboratory study to investigate the effects of the fly ash-based geopolymer content on the rheological and microstructural properties of asphalt binders. The results indicate that geopolymers could be used as a sustainable and greener alternative to other asphalt modifiers due to their low carbon dioxide footprint and better recycling capacity, having minor effects on the performance and microstructure of the binder.
The paper by M. Yu et al. presents an experimental study that evaluates the antiageing performance of recycled engine oil bottom (REOB) as a rejuvenating agent for asphalt binders and dense-graded asphalt mixtures. The main results demonstrate that REOB can be used as an asphalt regenerant, complying with the antiageing requirements reported in the technical specifications.

The work by Y. Xu et al. studies the effect of the blending degree between virgin and aged binder on the pavement performance of hot-mix recycled asphalt mixtures (HMRAM) with a high RAP content. To do this, HMRAMs with different degrees of blending (DOBs) were prepared by changing the mixing temperature. The results prove that the content and physical properties of old aggregates in RAP materials have an important effect on the performance of HMRAMs with a high RAP content.

In the paper by J. Li et al., similar to M. Yu et al., several physical and rheological tests were carried out in addition to four-component and infrared spectroscopic analyses on aged asphalt binders containing three types of REOB from a recycling plant. The main results of the paper reveal that $\mathrm{REOB}$ is sensitive to the softening point, and the asphaltene content has a significant effect on the physical and rheological properties of REOB-rejuvenated asphalt.

The paper by P. Hyzl et al. presents the results of a 5-year research focused on the use of reclaimed asphalt (RA) in asphalt mixtures of the type stone mastic asphalt (SMA). The authors determine that it is possible to use RA in SMA-type mixtures without negative impacts on the functional properties or on the layer lifetime. 
The paper by Y. Pi et al. analyses the general wetting process of asphalt on the aggregate surface based on the surface energy theory. As a main result, the authors establish a modified numerical wetting model to research the effects of initial temperature, cooling rate of asphalt, and aggregate surface texture sizes on the wetting process.

H. Li et al. study the effect of fly ash and slag on the properties of cement asphalt mortar (CAM). Experimental tests were conducted to determine the effect of these admixtures on the fresh and hardened characteristics of CAM. Based on the assessed properties, the CAM mixture with fly ash is suggested to be applied in further research as a costeffective strategy.

The work by D. Wang et al. examines the effect of chopped basalt fibres with different lengths and contents of addition on the performance of porous asphalt mixtures. The results prove that the addition of chopped basalt fibres can improve the performance of porous asphalt mixtures because they form a three-dimensional network reinforcing structure inside the porous asphalt mixture.

The paper by M. Pasetto et al. carries out an experimental study aimed at analysing the chromatic and mechanical properties of a clear synthetic resin and its suitability as an unconventional bituminous binder for novel asphalt mixtures. The studied synthetic resin was a proprietary product composed by a thermoplastic bicomponent resin with bonding properties like those of traditional bitumen. The binder developed shows promising results in view of its effective use in more sustainable asphalt pavements.

S. Magniafico et al. in their work study the differences between experimental linear viscoelastic (LVE) properties of both straight-run bitumen and a bituminous mixture and simulations with analogical 2S2P1D model fitted by 14 different users. 2S2P1D (2 springs, 2 parabolic creep elements, and 1 dashpot) is a linear viscoelastic analogical model to simulate the behaviour of bituminous materials developed at the University of Lyon/ENTPE. The obtained results highlight the relevance of 2 S2P1D model to perform multiscale modelling of LVE behaviour of bituminous materials, from bituminous binders to asphalt mixtures.

The paper by M. Sol-Sánchez et al. presents a research study that aims to analyse the reuse of zeolite waste derived from petroleum refining in the production of warm mix asphalts (WMA). Two different types of zeolite waste are analysed as additives for the manufacturing of two WMA, whose mechanical performance is compared with conventional WMA and hot-mix asphalt (HMA). The results indicate that zeolite waste with a content of $0.3 \%$ allows the production of WMA at temperatures around $145^{\circ} \mathrm{C}$, with comparable workability and densification to conventional reference HMAs at $165^{\circ} \mathrm{C}$, without reducing their mechanical properties.

The paper by H. Li et al. studies the induction heating efficiency and the healing capability of asphalt mixtures with different conductive additives. Steel fibre, steel grit, and steel slag are added to asphalt mixtures with induction heating purposes. The results show that the thermal properties of asphalt mixtures changed with the addition of different conductive additives. The modified asphalt mixture with steel fibre showed the best induction heating performance. It was proven that the addition of conductive additives in asphalt is a potential solution in order to propose novel asphalt pavements with crack-healing properties.

Finally, this special issue closes with the paper by D. Wang et al. They conduct an experimental and numerical study to establish a fatigue damage evolution model for the asphalt mixtures. To achieve this, four-point bending fatigue tests and FEM simulations by ABAQUS on test specimens of asphalt mixtures are performed. Based on the combination of the damage model by fatigue bending tests and their virtual simulation, the factors influencing the fatigue resistance of the whole asphalt mixtures are evaluated.

\section{Conflicts of Interest}

The editors declare that they have no conflicts of interest.

\section{Acknowledgments}

We would like to express our gratitude to all authors who made this special issue possible. We hope this collection of papers will be useful to the scientific community focused on novel sustainable bituminous materials.

José Norambuena-Contreras
Lily Poulikakos
Hassan Baaj
Quantao Liu 